\title{
Making Endovascular Neurosurgery Safer: Role of the Neuroanesthesiologist
}

\author{
Megha Uppal Sharma ${ }^{2}$ Pragati Ganjoo ${ }^{2}$ Sachin Jain ${ }^{3}$ \\ ${ }^{1}$ Department of Anaesthesiology, Artemis Hospital, Gurugram, \\ Haryana, India \\ ${ }^{2}$ Department of Anaesthesiology and Intensive Care, GB Pant \\ Institute of Postgraduate Medical Education and Research (GIPMER) \\ and Maulana Azad Medical College, New Delhi, India \\ ${ }^{3}$ Department of Neuroanaesthesia, Fortis Hospital, Noida, \\ Uttar Pradesh, India
}

\begin{abstract}
Address for correspondence Pragati Ganjoo, DA, DNB Anaesthesia, Department of Anaesthesiology and Intensive Care, GB Pant Institute of Postgraduate Medical Education and Research (GIPMER) and Maulana Azad Medical College, Jawaharlal Nehru Marg, New Delhi, India 110002 (e-mail: pganjoo@gmail.com).
\end{abstract}

J Neuroanaesthesiol Crit Care 2018;5:133-140
Abstract
Keywords
- endovascular neurosurgery
- interventional neuroradiology
- neuroendovascular procedures
- anesthesia
- perioperative complications

The increasing use of endovascular technique for treating neurovascular diseases underscores the need for the neuroanesthesiologist to be aware of its potentially serious perioperative complications that can directly impact the anesthetic outcome. These unique complications, including intracranial thromboembolism, hemorrhage, and vasospasm, and significant non-neurological complications that necessitate deft handling by experienced anesthesiologists. However, anesthesia for endovascular neurosurgery is generally being managed in remote hospital locations by relatively junior anesthesiologists who are likely to underestimate the importance of these closedcranium procedures. This review reacquaints the anesthetist with the periprocedural complications of endovascular neurosurgery that would enable their anticipation, prompt diagnosis, and effective management.

\section{Introduction}

The endovascular technique to diagnose and treat neurovascular lesions, known variously as endovascular neurosurgery or neuroendovascular procedures or interventional neuroradiology, involves fluoroscopy-guided administration of therapeutic drugs and devices into the cranial and spinal circulation. The common procedures include diagnostic angiography, coiling and stenting of cerebral aneurysms, glue embolization of cerebral arteriovenous malformations (AVMs), embolization of carotid-cavernous fistulae (CCF), embolization of intracranial tumors, balloon angioplasty for treating vasospasm and atherosclerosis, and combined angioplasty and carotid artery stenting for carotid artery stenosis (CAS), intra-arterial (IA) vasodilator administration, sclerotherapy of venous angiomas, percutaneous transluminal angioplasty and stenting (PTAS) for treating intracranial stenosis and intracranial/extracranial atherosclerosis, and treatment of thromboembolic strokes with thrombolysis and thrombectomy. ${ }^{1-10}$ Better outcomes with endovascular coiling compared with craniotomy and clipping were reported by The International Subarachnoid Aneurysm Trial (ISAT) 2002 and the Cochrane collaboration particularly in anterior circulation aneurysms in good grade subarachnoid hemorrhage (SAH) patients..$^{11,12}$ Molyneux et al have reinforced these findings in the ISAT follow-up study published in 2015. They have demonstrated that for patients with suitable aneurysms, coiling is more likely than clipping to result in improved clinical outcomes at 1 year, and the early clinical benefits are not lost over the subsequent years. They have demonstrated that although rates of increased dependency did not differ, the probability of death or dependency was significantly greater in clipping versus coiling group. Rebleeding was more likely after endovascular coiling compared from clipping. However, this risk was small, and the probability of disability-free survival was significantly greater in the endovascular group than in the neurosurgical group at 10 -year follow-up. ${ }^{13}$

The increasing use of the endovascular approach to treat complex neurosurgical lesions such as aneurysms and AVMs has introduced newer challenges for the neuroanesthesiologists. However, endovascular neurosurgery is not getting the same anesthetic attention as the traditional open craniotomy neurosurgery in many centers. As there is no overt bleeding

Copyright $\odot 2018$ Indian Society of Neuroanaesthesiology and Critical Care
License terms

() (1) $\circledast$ 
or ill-effects of brain/spine retraction and handling in endovascular procedures, many novice anesthesiologists consider them to be simple and innocuous procedures, and the anesthetic management is often left to relatively inexperienced and less knowledgeable junior staff. However, endovascular neurosurgery, like its open counterpart, is also associated with potentially serious complications resulting in significant patient morbidity and mortality. These complications, generated by the unique endovascular techniques, include aneurysm rupture, thromboembolism, intracranial vessel injury or dissection, coil displacement or fracture causing parent artery compromise, vasospasm, cerebral edema, hemorrhage and ischemia, contrast reactions, contrast nephropathy, groin hemorrhage or hematoma, hypothermia, and fluid-electrolyte imbalance. The adverse consequences of these complications, such as hemodynamic instability, respiratory insufficiency, delayed awakening, and post-procedure neurological deterioration, seizures, stroke, and death can significantly impact the anesthetic course and outcome ${ }^{1,2} ; \mathrm{a}$ good understanding of these complications is thus imperative for the concerned anesthetists. This review primarily focuses on the periprocedural complications of neuroendovascular procedures and highlights the role of the anesthetist in their effective management in coordination with the interventionist that would help improve the safety of these procedures.

\section{An Overview of the Procedure Technique and Anesthesia Protocol}

The neuroendovascular procedure involves digital subtraction angiography and road mapping using nonionized contrast (ioxehol) under high-resolution fluoroscopy, followed by transfemoral artery catheterization by the Seldinger technique. Protection against radiation hazards is mandatory for all operating room personnel. The femoral artery is punctured by a 6-8 Fr check-flow cannula, and through this, a 5-7 Fr end-hole guiding catheter is negotiated into the feeder artery of the vascular lesion. Further, super-selective catheterization of the vascular lesions is done using 1-2.4 Fr microcatheters. Continuous irrigation of the catheters is done by heparinized normal saline infusion delivered at 200 to $250 \mathrm{~mm} \mathrm{Hg}$ pressure. Through the microcatheters, drugs and devices such as coils, polyvinyl alcohol (PVA) particles, Onyx, N-butyl cyanoacrylate, and revascularization stenting devices are delivered into the lesion. ${ }^{6,10,14,15}$ The coil deployment methods can be electrical (Guglielmi detachable coils), mechanical (Cook coils), or thermal (Micrus endovascular coils). ${ }^{6,16,17}$ Stent-assisted coil delivery may be used in acutely ruptured aneurysms ${ }^{18}$; flow-diverting stents are promising for the treatment of wide-necked, large, or giant aneurysms or multiple aneurysms of the internal carotid artery (ICA).,19 The patient is anticoagulated during the procedure with intravenous (IV) heparin boluses (60-70 IU/ $\mathrm{kg}$ initially at the time of check-flow insertion and one-third initial dose repeated hourly) to maintain the activated clotting time (ACT) at two to three times the baseline values. The catheter irrigation infusion contains 1,000 IU heparin/L of normal saline. The check-flow sheath is removed only when the post-procedure ACT normalizes, and the cannulation site is manually pressed till bleeding stops.

The anesthetic management includes providing a welltitrated, procedure-specific anesthesia, prompt management of the sudden and serious periprocedural complications in the usually remotely located and inadequately equipped neuroradiology suites, and safe intra-hospital transport of the critically ill patients between these distant locations and the neurointensive care units (NICUs). Anesthesia is directed toward maintaining a good hemodynamic control, manipulating systemic and cerebral blood flows in accordance with the procedure, managing anticoagulation, ensuring intra-procedural patient immobility while ensuring rapid awakening at the end to facilitate early neurological assessment, quickly diagnosing procedure-related complications, and instituting early neuroprotective measures to limit the intracerebral damage. ${ }^{1,2}$ Specific preanesthetic considerations include ruling out pregnancy and co-morbidities, such as renal disease, diabetes, and allergy to iodine and protamine, ${ }^{20}$ and evaluation of the coagulation profile including ACT and serum electrolytes. Neurological evaluation of the patient should be prior to the procedure using Glasgow Coma Scale (GCS). Patients with poor neurological grade may require preprocedure ventilation, and those with cerebral vasospasm may be started on IV nimodipine infusion at $2 \mathrm{mg} / \mathrm{h}$. Patients posted for flow-diverting stents or stent-assisted coil embolization for unruptured aneurysms may be considered for pre-procedure therapy with aspirin, heparin, and dual antiplatelet drugs, ${ }^{21-23}$ though its benefit should be balanced with the increased risk of intracranial bleeding.,24 The procedures can be performed under either general anesthesia (GA) or conscious sedation. GA provides patient immobility, better image quality, and the ability to easily titrate systemic and cerebral perfusion. ${ }^{25,26}$ Target-controlled infusion of propofol and fentanyl/remifentanil provides cardiovascular stability, easy titration of blood pressure, and rapid emergence. ${ }^{5}$ Total intravenous anesthesia (TIVA) with propofol is advantageous as cerebral vasomotor tone is preserved, and improved flow metabolism coupling and carbon dioxide responsiveness is achieved with less vasodilatation and a lower intracranial pressure (ICP). ${ }^{25}$ If volatile agents are chosen, sevoflurane and desflurane are the agents of choice. ${ }^{27}$ Choice of anesthetic technique for GA varies according to institutional practices; any anesthetic drug combination that can be easily titrated can be used. Conscious sedation helps in early diagnosis of intra-procedural neurological deterioration and angina in the awake patient and is preferred for procedures such as diagnostic angiography, carotid artery stenting, PTAS, and stroke management. ${ }^{28-33}$ Proponents of sedation for endovascular management of stroke suggest that even mild hypotension due to GA may result in the rapid conversion of the ischemic penumbra into infarction. ${ }^{34}$ The theoretical concern that hyperextension and manipulations of the neck during induction and intubation could cause kinking of the carotid or vertebral arteries compromising cerebral perfusion has also been raised. ${ }^{26}$ Ability to continuously assess neurological status when the patient has not been anesthetized may 
allow determining the endpoint of the procedure clinically rather than angiographically. Furthermore, severe persistent pain may herald vessel rupture or dissection and thus prewarn the interventionist to modify the technique to avoid a catastrophe. ${ }^{26}$ However, these patients may urgently need to be converted to GA when there is sudden neurological deterioration or over-sedation. It should be noted that for patients taken up under IV sedation, injection of contrast can cause traction of cerebral vasculature, resulting in burning sensation and headache. Placement of a nasopharyngeal airway during administering sedation may result in inadvertent bleeding in the anticoagulated patient undergoing neurointervention. Dexmedetomidine is gaining popularity for IV sedation in view of its lack of respiratory depression and easy arousability of the patient that allows early assessment of neurological function after completion of the procedure. However, it may cause hypotension that can persist post-procedure, and its effects on cerebral perfusion are still unknown; this may decrease its applicability in interventional neuroradiology. 5,25

Specific monitoring during these procedures includes the ACT, fluid intake-output, serial arterial blood gases, serum electrolytes, and close watch on the C-arm monitor screen for early detection of complications; somatosensory-evoked potential and motor evoked-potential monitoring may be required for embolization of spinal AVMs. Forced-air blankets and pre-heated IV fluids, drug infusions, and irrigation fluids are recommended for maintaining normothermia. Anesthesia is mostly reversed on table, and the patients are closely monitored in the NICU. Deliberate hypotension (arterial blood pressure [ABP] 15 to $20 \%$ below baseline) is used in post-AVM embolization patients, ${ }^{35-37}$ while deliberate hypertension (ABP 15 to $20 \%$ above baseline) is used after aneurysm coiling. Nimodipine infusion is given at $2 \mathrm{mg} / \mathrm{h}$ for the initial 48 hours following aneurysm coiling and later continued orally at $60 \mathrm{mg}$, 4 hourly doses for 5 to 7 days.

\section{Complications of Neuroendovascular Procedures}

The complications occur inside a closed cranium, and hence, can be catastrophic. As overt clinical manifestations are few, anticipation of the complications at each step and vigilant monitoring enable their prompt diagnosis and management. The initial management is done by the interventionist, usually by rapidly modifying the procedure there itself. The neuroanesthesiologist's role is vital in limiting the severity of complications by managing anticoagulation, maintaining systemic and cerebral hemodynamic stability, and instituting early neuroprotective measures. A good understanding and close coordination are thus necessary between the interventionist and the anesthetist.

\section{Hemorrhagic Complications}

Intracranial hemorrhage is a dreaded complication of neuroendovascular procedures and can occur due to aneurysm rupture or perforation or dissection of major blood vessels. Stent-assisted coil delivery has a higher risk of complications and death in comparison to therapy without stent placement, ${ }^{18}$ and delayed aneurysm rupture is more common after therapy with flow-diverting stents. ${ }^{3,19}$ In sedated patients, hemorrhage manifests with headache, nausea, vomiting, and vascular pain related to the area of perforation. In the anesthetized patients, it is suspected if extravasation of contrast is seen during fluoroscopy, which may be accompanied by hemodynamic alterations such as abrupt bradycardia and hypertension, widened pulse pressure, tachycardia, and arrhythmias. Aneurysms may rupture during microcatheter negotiation, coil delivery, or blood pressure surges, especially at induction of GA. Manipulation of guidewires or catheters can induce perforation/dissection of the feeding arteries. Management of aneurysm rupture is done with immediate lowering of ABP by increasing the depth of anesthesia or using anti-hypertensives such as IV labetalol, securing the airway in sedated patients, ventilation with $100 \%$ oxygen $\left(\mathrm{O}_{2}\right)$, maintaining hypocapnia, and administration of IV mannitol and anticonvulsants. Reversal of heparin action is done with protamine (1:1 ratio). The interventionist rapidly packs the ruptured aneurysm with additional coils, and if that fails, emergency craniotomy and aneurysm clipping may be considered, though this is uncommon ${ }^{5,6,38}$; external ventriculostomy is required if acute hydrocephalus develops. In case of a sudden massive intracranial catastrophe, deliberate hypothermia $\left(33-34^{\circ} \mathrm{C}\right)$, hyperventilation and electroencephalography burst suppression using IV anesthetic agents has also been suggested. ${ }^{7}$ Post-procedure ventilation following aneurysm rupture helps to manage adverse consequences such as cerebral edema, hematoma, and acute hydrocephalus. Small vessel perforations may be treated conservatively or the catheter itself may be used to occlude the perforation. The reported incidence of aneurysm rupture varies between 1.69 and 8.8\%. ${ }^{39-45}$ Hemorrhage can also occur at the femoral sheath insertion site due to inadequate groin compression after sheath removal. Dissection of femoral artery or a retroperitoneal hematoma formation are other possible hemorrhagic complications. Hyperemic complications in AVM embolization can result in cerebral hemorrhage and edema during or after the procedure. It may occur secondary to "normal perfusion pressure breakthrough (NPPB) hyperemia" or to "occlusive hyperemia" due to venous thrombosis. ${ }^{46,47}$ The NPPB syndrome occurs due to abrupt restoration of normal systemic pressure to a chronically hypotensive vascular bed that can overwhelm its autoregulatory capacity and result in hemorrhage or swelling. Maintaining a good intra-procedure $\mathrm{ABP}$ control is thus necessary during neuroendovascular procedures.

\section{Occlusive Complications}

Thromboembolism, vasospasm, coil displacement causing parent artery compromise (incidence: 2.9\%), and coil fracture are among the occlusive complications causing serious post-procedure problems such as thromboembolic stroke, cerebral ischemia/infarction, cerebral edema, and death. ${ }^{6,41}$ Despite systemic heparinization, thrombi can develop, either during guidewire manipulations inside the catheter or during insertion and withdrawal of coils and stents. Disruption of an 
atherosclerotic plaque by the catheter or guidewire or rare coagulation disorders such as heparin-induced thrombocytopenia (HIT) and antiphospholipid antibody syndrome can also cause occlusive complications. ${ }^{48}$ Quick-acting glues and particulate material used for AVM embolization can pass into the systemic circulation causing inadvertent embolization of the normal brain vasculature ${ }^{8}$; embolization of the solvent dimethyl sulfoxide to lung can cause pulmonary edema. ${ }^{49}$ Prevention of occlusive complications can be done by minimizing guidewire manipulations and continuous catheter flushing with heparinized saline. Treatment includes raising the mean ABP by 30 to $40 \%$ above baseline (deliberate hypertension), maintaining normocarbia, and institution of urgent neuroprotection methods. Mechanical lysis of angiographically visible thrombi can be done with mechanical devices or saline infusion or with the use of thrombolytic agents such as local tissue plasminogen activator or local and IV antiplatelet agent Abciximab (GPIIb/IIIa inhibitor). IV aspirin has also been administered. ${ }^{2}$ Malpositioned coils compromising the parent artery can be retrieved or repositioned by the endovascular method itself, and if it fails, craniotomy is required. Aneurysm coiling is reported to have a 1.69 to $5 \%$ incidence of thromboembolic complications, while AVM embolization has a 1 to $8 \%$ incidence of potentially fatal ischemic/hemorrhagic complications. ${ }^{6,14,40-43,50}$

\section{Vasospasm}

Vasospasm is a common complication after neuroendovascular procedures that may be responsible for delayed awakening from anesthesia and post-procedure neurological deterioration. The incidence of intra-procedure vasospasm in patients undergoing aneurysm coiling has been reported variously as $2.94,5.94,6.67,9.52$, and $9 \% 39,41,43,48,51$ and of post-procedure vasospasm as 6 and 7.08\%.43.48 Management of vasospasm has moved away from the traditional triple- $\mathrm{H}$ therapy (hypertension, hypervolemia, and hemodilution) toward maintaining hypertension and euvolemia. ${ }^{2}$ The systolic BP is aimed at 160 to $180 \mathrm{~mm} \mathrm{Hg}$ in secured aneurysms and 140 to $160 \mathrm{~mm} \mathrm{Hg}$ in unsecured aneurysms and can be achieved by IV phenylephrine., ${ }^{2,7}$ Deliberate hypertension helps increase the collateral perfusion pressure in the circle of Willis, pial-to-pial, and leptomeningeal collaterals. ${ }^{52}$ Earlier a central venous pressure (CVP) of 8 to $12 \mathrm{~mm} \mathrm{Hg}$, pulmonary artery wedge pressure of 15 to $18 \mathrm{~mm} \mathrm{Hg}$, and a hematocrit between 0.3 and 0.35 along with hypertension (triple- $\mathrm{H}$ therapy) were recommended. ${ }^{33}$ However, as triple-H therapy had major side effects such as pulmonary edema, myocardial ischemia, electrolyte imbalance, and cerebral edema, it is no longer recommended. ${ }^{54,55}$ Pharmacologic angioplasty with intra-arterial (IA) papaverine and IV nimodipine, nicardipine, and verapamil has shown clinical improvement; however, papaverine effect is transient, and it may cause monocular blindness, mydriasis, seizures, transient increase in ICP, hypertension, tachycardia, and paradoxical worsening of vasospasm. ${ }^{56}$ Mechanical (balloon) angioplasty, if performed within 2 hours of symptomatic ischemia, is an effective treatment method for vasospasm. ., $654-56^{-6}$

\section{Hemodynamic Perturbations}

Close hemodynamic monitoring aids early detection of periprocedural complications. While transient bradycardia, hypotension, and even asystole may be expected in carotid stenting procedures, any other abrupt periprocedure hemodynamic alterations may signify development of serious complications such as intracranial hemorrhage or raised ICP. ${ }^{57-60}$ Hypertension with tachycardia may herald aneurysm rupture, but subsequently, there may be hypotension. Reported incidence of hemodynamic perturbations during aneurysm coiling ranges from 4 to $7.9 \%{ }^{39,43,48}$ Management is by stopping the stimulating maneuvers till the vital parameters stabilize, treatment with cardiac drugs and fluids, and correction of the underlying cause. In patients with contralateral ICA occlusion, severe vertebrobasilar disease, or additional intracranial stenosis, hypotension can be particularly detrimental and pre-procedure placement of transcutaneous pacing leads may be considered.

\section{Non-neurological Complications}

Patients with SAH are already prone to develop electrolyte abnormalities such as hyponatremia, hypokalemia, hypocalcemia, and hypomagnesemia secondary to inappropriate antidiuretic hormone secretion or cerebral salt wasting syndrome. ${ }^{5,61}$ Electrolyte imbalance and fluid overload can further occur during neuroendovascular procedures due to the large volume of irrigation fluids administered; the geriatric and cardiac patients are especially prone to develop deleterious cardiac and respiratory consequences. Though CVP monitoring for managing intra-procedure fluid therapy is not mandatory in endovascular procedures, its use may benefit the vulnerable population. Coagulation complications can occur due to mismanagement of heparin dosing leading to cerebral thromboembolism or hemorrhage; periprocedure serial ACT monitoring is hence valuable. Argatroban, a direct thrombin inhibitor, has shown promise in patients predisposed to HIT.62 The administration of contrast agents can cause contrast reactions and nephropathy. Risk factors for contrast nephropathy include diabetes mellitus, pre-existing renal disease, co-administration of nephrotoxic drugs, ionic contrast, volume depletion, and high osmolality or high dose of contrast. Maintenance of normovolemia is imperative as contrast agents have diuretic effects. Fatal contrast reactions are known and can be prevented by using nonionic contrasts such as iohexol and pretreatment of predisposed patients with antihistamines and steroids. N-acetylcysteine, 600 to 1,200 $\mathrm{mg}$ twice daily, on the day prior to the procedure and on the day of procedure (total 2 days) is acceptable prophylaxis in high-risk patients. ${ }^{63}$ Isotonic bicarbonate infusion can reduce the incidence of contrast-induced nephropathy by alkalizing renal tubular fluid and reducing tubular damage. ${ }^{20,64}$ Hypothermia is a known problem during endovascular procedures due to the low ambient temperatures of the neuroradiology suites, continuous infusion of large volume of fluid, and inadequate patient cover because of groin exposure. Management is by using pre-warmed fluids and heated patient mattresses. Delayed awakening of patients after the procedure can occur 
due to hypothermia, electrolyte abnormalities, or intra-procedure neurological complications. Delayed emergence was reported in $2 \%$ patients secondary to intracranial bleeding. ${ }^{45}$ Patients with neurological insults such as aneurysm rupture, severe vasospasm, or thromboembolic episodes may require elective post-procedure ventilation. Early post-procedure problems include nausea, vomiting, groin hemorrhage/hematoma, fluid-electrolyte imbalance, pulmonary edema, osmotic diuresis leading to dehydration, vasospasm leading to neurological deterioration, transient ischemic attacks, stroke, seizures, infection/meningitis, and even death. ${ }^{6,43}$

\section{Radiation Hazards}

Exposure to ionizing radiation in high doses is an occupational hazard of endovascular procedures and results in late complications in the patient as well as the medical personnel. Direct radiation from the X-ray tube, leaked radiation through the protective gear, and radiation exposure from scatter contribute to the radiation exposure. The International Commission on Radiological Protection (ICRP) recommends that the radiation exposure should not exceed $20 \mathrm{mSv} /$ year, averaged over 5 years, with no more than $50 \mathrm{mSv}$ in any 1 year. Exposure to the eye should not exceed $150 \mathrm{mSv} /$ year. ${ }^{65}$ In the United States, it is implemented as an annual occupational limit of $50 \mathrm{mSv}$ and a lifetime limit of $10 \mathrm{mSv}$ multiplied by the age in years ${ }^{66}$ If protective gear is routinely used and safety precautions are followed, the exposure usually does not exceed the safety limits. The protective gear includes lead aprons that cover the front and back of body and lead thyroid shields consisting of lead equivalents of $0.35 \mathrm{~mm} \mathrm{~Pb}$, and portable lead acrylic shields. Radiation exposure is directly proportional to the time of exposure and indirectly proportional to the square of the distance from the radiation source, and hence, the duration of exposure should be minimized and the distance from the source maximized. ${ }^{67}$ Stationing of personnel at least 4 feet away from the radiation source, operating the fluoroscope at the lowest dose rate that yields adequate images, and monitoring the radiation time have been suggested to minimize radiation hazards.,61 Standing on the receiver side of the C-Arm to minimize scatter from the surface of the patient facing the radiation source is also suggested. ${ }^{67}$

\section{Expanding Horizons of Neuroendovascular Procedures}

With rapidly evolving neuroendovascular technology, newer indications and procedures are on the rise, which however, bring in their own set of complications. Endovascular balloon angioplasty with stenting has emerged as an effective alternative to carotid endarterectomy for the treatment of CAS. ${ }^{27}$ There is a decreased risk of periprocedural myocardial ischemia with carotid stenting compared with carotid endarterectomy, but it is associated with a higher risk of stroke. ${ }^{55,57,68}$ Intra-procedural transient bradycardia and hypotension due to carotid body stimulation is well known; older patients with heavily calcified lesions may have prolonged, severe hypotension. Prophylactic administration of atropine and placement of temporary cardiac pacing wires should be considered, and an external defibrillator should be kept on standby; hypotension can be managed with IV fluids and vasopressors. Careful hemodynamic monitoring in the first 24 hours following carotid stenting is thus very crucial. ${ }^{69,70}$ Distal thromboembolism, periprocedural stroke, myocardial ischemia due to hypertension, cerebral hyper-perfusion syndrome, and vocal cord paralysis can also occur. Intra-procedural spasm of the distal ICA can be catastrophic if the contralateral artery is also compromised; IA administration of 100 to $400 \mu \mathrm{g}$ of nitroglycerine by the interventionist generally leads to a rapid resolution of spasm. PTAS is increasingly being used for treating intracranial stenosis exceeding 50\% and stenosis with recent-onset symptoms despite optimal medical treatment; the self-expanding bare metal WINGSPAN stent is the most commonly used device with a reported technical success rate of $98 \%$. However, at present, recommendations for stenting in intracranial stenosis are still under evaluation. ${ }^{71}$ The anesthetist needs to be alert for onset of sudden intra-procedural apnea during endovascular treatment of basilar artery stenosis. ${ }^{32}$ Craniofacial malformations with intracranial vascular connections can present for sclerotherapy under fluoroscopy guidance; ethanol (95\%) opacified with contrast is the commonly used agent but needs to be used cautiously. Ethanol injection can cause marked airway swelling leading to sudden hypoxia. Nerve damage, trismus, agitation after emergence from anesthesia, renal toxicity, hepatotoxicity, and even cardiopulmonary arrest due to ethanol intoxication are possible complications..$^{72}$ The embolic/sclerosing agents can move into the cerebral veins via the draining dural fistulae and craniofacial vascular malformations; increasing the cerebral venous outflow above the extracranial venous outflow by deliberate hypercapnia (partial pressure of carbon dioxide $\left[\mathrm{PaCO}_{2}\right]$ of $50-60 \mathrm{~mm} \mathrm{Hg}$ ) may be required to prevent this intracerebral movement. IA stroke thrombolysis is also on the rise and is indicated in major middle cerebral artery strokes within 6 hours in patients not suitable for IV thrombolysis ${ }^{73}$; mechanical thrombectomy can be used as an adjunct to IA/ IV thrombolysis or as first-line treatment for large vessel occlusive stroke. ${ }^{74}$ The procedure is best done under sedation, and hypotension (systolic blood pressure (SBP) $<140$ $\mathrm{mm} \mathrm{Hg}$ ) and hyperthermia (temperature $>37.2^{\circ} \mathrm{C}$ ) should be avoided..$^{34,72,75-77}$ These procedures are associated with an elevated risk of intracerebral hemorrhage.

\section{Conclusion}

To summarize, administering anesthesia and managing the distinctive complications of neuroendovascular procedures can be challenging due to the unique condition of a closed cranium. The remote location of the neuroradiology suite with a small working area, inadequate monitoring, distant help, and poor resuscitation facilities add to the woes of a beginner anesthesiologist. ${ }^{78,79}$ Having experienced and dedicated neuro-anesthetists for these procedures, promoting a good understanding and communication between the neurointerventional and neuroanesthesia teams, and placing the neuroradiology suites closer to the neurosurgical operating area and NICU can go a long way in achieving favorable results. 


\author{
Funding \\ None.

\section{Conflict of Interest} \\ None.
}

\section{References}

1 Jansen O, Szikora I, Causin F, Brückmann H, Lobotesis K. Standards of practice in interventional neuroradiology. Neuroradiology 2017;59(6):541-544

2 Patel S, Reddy U. Anaesthesia for interventional neuroradiology. BJA Educ 2016;16(5):147-152

3 Ferrell AS, Britz GW. Developments on the horizon in the treatment of neurovascular problems. Surg Neurol Int 2013;4(Suppl 1):S31-S37

4 Hashimoto T, Gupta DK, Young WL. Interventional neuroradiology--anesthetic considerations. Anesthesiol Clin North America 2002;20(2):347-359, vi

5 Schulenburg E, Matta B. Anaesthesia for interventional neuroradiology. Curr Opin Anaesthesiol 2011;24(4):426-432

6 Varma MK, Price K, Jayakrishnan V, Manickam B, Kessell G. Anaesthetic considerations for interventional neuroradiology. Br J Anaesth 2007;99(1):75-85

7 Lee CZ, Young WL. Anesthesia for endovascular neurosurgery and interventional neuroradiology. Anesthesiol Clin 2012;30(2):127-147

8 Osborn IP. Anesthetic considerations for interventional neuroradiology. Int Anesthesiol Clin 2003;41(2):69-77

9 Dorairaj IL, Hancock SM. Anaesthesia for interventional neuroradiology. Contin Educ Anaesth Crit Care Pain 2008;8:86-89

10 Sinha PK, Neema PK, Rathod RC. Anesthesia and intracranial arteriovenous malformation. Neurol India 2004;52(2):163-170

11 Molyneux A, Kerr R, Stratton I, et al; International Subarachnoid Aneurysm Trial (ISAT) Collaborative Group. International Subarachnoid Aneurysm Trial (ISAT) of neurosurgical clipping versus endovascular coiling in 2143 patients with ruptured intracranial aneurysms: a randomised trial. Lancet 2002;360(9342):1267-1274

12 van der Schaaf I, Algra A, Wermer M, et al. Endovascular coiling versus neurosurgical clipping for patients with aneurysmal subarachnoid haemorrhage. Cochrane Database Syst Rev 2005;4(4):CD003085

13 Molyneux AJ, Birks J, Clarke A, Sneade M, Kerr RSC. The durability of endovascular coiling versus neurosurgical clipping of ruptured cerebral aneurysms: 18 year follow-up of the UK cohort of the International Subarachnoid Aneurysm Trial (ISAT) Lancet 2015;385(9969):691-697

14 Martin NA, Khanna R, Doberstein C, Bentson J. Therapeutic embolization of arteriovenous malformations: the case for and against. Clin Neurosurg 2000;46:295-318

15 Miller C, Mirski M. Anesthesia considerations and intraoperative monitoring during surgery for arteriovenous malformations and dural arteriovenous fistulas. Neurosurg Clin $\mathrm{N} \mathrm{Am}$ 2012;23(1):153-164

16 Guglielmi G, Viñuela F, Sepetka I, Macellari V. Electrothrombosis of saccular aneurysms via endovascular approach. Part 1: Electrochemical basis, technique, and experimental results. J Neurosurg 1991;75(1):1-7

17 Guglielmi G, Viñuela F, Dion J, Duckwiler G. Electrothrombosis of saccular aneurysms via endovascular approach. Part 2: Preliminary clinical experience. J Neurosurg 1991;75(1):8-14

18 Bodily KD, Cloft HJ, Lanzino G, Fiorella DJ, White PM, Kallmes DF. Stent-assisted coiling in acutely ruptured intracranial aneurysms: a qualitative, systematic review of the literature. Am J Neuroradiol 2011;32(7):1232-1236
19 Siddiqui AH, Abla AA, Kan P, et al. Panacea or problem: flow diverters in the treatment of symptomatic large or giant fusiform vertebrobasilar aneurysms. J Neurosurg 2012;116(6):1258-1266

20 Murphy SW, Barrett BJ, Parfrey PS. Contrast nephropathy. J Am Soc Nephrol 2000;11(1):177-182

21 Sedat J, Chau Y, Gaudart J, Sachet M, Beuil S, Lonjon M. Prasugrel versus clopidogrel in stent-assisted coil embolization of unruptured intracranial aneurysms. Interv Neuroradiol 2017;23(1):52-59

22 Jahshan S, Abla AA, Natarajan SK, et al. Results of stent-assisted vs non-stent-assisted endovascular therapies in 489 cerebral aneurysms: single-center experience. Neurosurgery 2013;72(2):232-239

23 Shapiro M, Becske T, Sahlein D, Babb J, Nelson PK. Stent-supported aneurysm coiling: a literature survey of treatment and follow-up. Am J Neuroradiol 2012;33(1):159-163

24 Tumialán LM, Zhang YJ, Cawley CM, Dion JE, Tong FC, Barrow DL. Intracranial hemorrhage associated with stent-assisted coil embolization of cerebral aneurysms: a cautionary report. J Neurosurg 2008;108(6):1122-1129

25 Hans P, Bonhomme V. Why we still use intravenous drugs as the basic regimen for neurosurgical anaesthesia. Curr Opin Anaesthesiol 2006;19(5):498-503

26 Molina CA, Selim MH. General or local anesthesia during endovascular procedures: sailing quiet in the darkness or fast under a daylight storm. Stroke 2010;41(11):2720-2721

27 Erickson KM, Cole DJ. Carotid artery disease: stenting vs endarterectomy. Br J Anaesth 2010;105(Suppl 1):i34-i49

28 Brinjikji W, Pasternak J, Murad MH, et al. Anesthesia-related outcomes for endovascular stroke revascularisation. A systematic review and meta-analysis. Stroke 2017;48(10):2784-2791

29 Zussman B, Weiner G, Ducruet A. A second randomized trial comparing general anesthesia to conscious sedation in acute ischemic stroke patients undergoing endovascular treatment. Neurosurgery 2018;82(3):N27-N28

30 Pfaff JAR, Schönenberger S, Nagel S, et al. Effect of general anesthesia versus conscious sedation for stroke thrombectomy on angiographic workflow in a randomized trial: a post hoc analysis of the SIESTA trial. Radiology 2018;286(3):1016-1021

31 Campbell BCV, van Zwam WH, Goyal M, et al; HERMES collaborators. Effect of general anaesthesia on functional outcome in patients with anterior circulation ischaemic stroke having endovascular thrombectomy versus standard care: a meta-analysis of individual patient data. Lancet Neurol 2018;17(1):47-53

32 Reddy U, Smith M. Anesthetic management of endovascular procedures for cerebrovascular atherosclerosis. Curr Opin Anaesthesiol 2012;25(4):486-492

33 Abou-Chebl A, Lin R, Hussain MS, et al. Conscious sedation versus general anesthesia during endovascular therapy for acute anterior circulation stroke: preliminary results from a retrospective, multicenter study. Stroke 2010;41(6):1175-1179

34 Delgado-Mederos R, Ribo M, Rovira A, et al. Prognostic significance of blood pressure variability after thrombolysis in acute stroke. Neurology 2008;71(8):552-558

35 Szabo MD, Crosby G, Sundaram P, Dodson BA, Kjellberg RN. Hypertension does not cause spontaneous hemorrhage of intracranial arteriovenous malformations. Anesthesiology 1989;70(5):761-763

36 Yakes WF, Rossi P, Odink H. How I do it. Arteriovenous malformation management. Cardiovasc Intervent Radiol 1996;19(2):65-71

37 Hashimoto T, Young WL, Aagaard BD, Joshi S, Ostapkovich ND, Pile-Spellman J. Adenosine-induced ventricular asystole to induce transient profound systemic hypotension in patients undergoing endovascular therapy. Dose-response characteristics. Anesthesiology 2000;93(4):998-1001 
38 El Barbary MM, Zaki GFS, Mohamed MH, Mahmoud NM. Anesthetic considerations for endo-vascular management of intracranial aneurysms. Egypt J Hosp Med 2017;69(2):1864-1873

39 Asouhidou I, Asteri T, Katsaridis V, Georgiadis G. Immediate anesthesia management of complications during embolization of cerebral vascular lesions. The Internet Journal of Anesthesiology 2006;14(2)

40 Derbent A, Oran I, Parildar M, Yurtseven T, Uyar M, Memiş A. Adverse effects of anesthesia in interventional radiology. Diagn Interv Radiol 2005;11(2):109-112

41 Deng J, Zhao Z, Gao G. Periprocedural complications associated with endovascular embolisation of intracranial ruptured aneurysms with matrix coils. Singapore Med J 2007;48(5):429-433

42 Park SH, Lee CY, Yim MB. The merits of endovascular coil surgery for patients with unruptured intracranial aneurysms. J Korean Neurosurg Soc 2008;43(6):270-274

43 Sharma MU, Ganjoo P, Singh D, et al. Perioperative complications in endovascular neurosurgery: anesthesiologist's perspective. Asian J Neurosurg 2017;12(1):6-12

44 Sharma DP, Singh D, Jagetia A, Singh H, Tandon M, Ganjoo P. Intra procedure rupture of intracranial aneurysm during endovascular coiling: neurosurgeons' experience and review of the literature. Neurol India 2011;59(5):690-695

45 Lai YC, Manninen PH. Anesthesia for cerebral aneurysms: a comparison between interventional neuroradiology and surgery. Can J Anaesth 2001;48(4):391-395

46 Rangel-Castilla L, Spetzler RF, Nakaji P. Normal perfusion pressure breakthrough theory: a reappraisal after 35 years. Neurosurg Rev 2015;38(3):399-404, discussion 404-405

47 Zacharia BE, Bruce S, Appelboom G, Connolly ES Jr. Occlusive hyperemia versus normal perfusion pressure breakthrough after treatment of cranial arteriovenous malformations. Neurosurg Clin N Am 2012;23(1):147-151

48 Gupta MM, Bithal PK, Dash HH, Chaturvedi A, Prabhakar H. Clinical outcome of intracranial aneurysms: A retrospective comparison between endovascular coiling and neurosurgical clipping. Indian J Anaesth 2008;52(1):63-69

49 Carapiet DA, Stevens JE. Pulmonary embolism following embolization of an arteriovenous malformation. Paediatr Anaesth 1996;6(6):491-494

50 Lv X, Wu Z, Jiang C, et al. Complication risk of endovascular embolization for cerebral arteriovenous malformation. Eur J Radiol 2011;80(3):776-779

51 Rabinstein AA, Nichols DA. Endovascular coil embolization of cerebral aneurysm remnants after incomplete surgical obliteration. Stroke 2002;33(7):1809-1815

52 Treggiari MM; Participants in the International Multidisciplinary Consensus Conference on the Critical Care Management of Subarachnoid Hemorrhage. Hemodynamic management of subarachnoid hemorrhage. Neurocrit Care 2011;15(2):329-335

53 Priebe HJ. Aneurysmal subarachnoid haemorrhage and the anaesthetist. Br J Anaesth 2007;99(1):102-118

54 Egge A, Waterloo K, Sjøholm H, Solberg T, Ingebrigtsen T, Romner B. Prophylactic hyperdynamic postoperative fluid therapy after aneurysmal subarachnoid hemorrhage: a clinical, prospective, randomized, controlled study. Neurosurgery 2001;49(3):593-605, discussion 605-606

55 Treggiari-Venzi MM, Suter PM, Romand JA. Review of medical prevention of vasospasm after aneurysmal subarachnoid hemorrhage: a problem of neurointensive care. Neurosurgery 2001;48(2):249-261, discussion 261-262

56 Clyde BL, Firlik AD, Kaufmann AM, Spearman MP, Yonas H. Paradoxical aggravation of vasospasm with papaverine infusion following aneurysmal subarachnoid hemorrhage. Case report. J Neurosurg 1996;84(4):690-695
57 Yavin D, Roberts DJ, Tso M, Sutherland GR, Eliasziw M, Wong $\mathrm{JH}$. Carotid endarterectomy versus stenting: a meta-analysis of randomized trials. Can J Neurol Sci 2011;38(2):230-235

58 Siddiqui AH, Natarajan SK, Hopkins LN, Levy EI. Carotid artery stenting for primary and secondary stroke prevention. World Neurosurg 2011;76(6, Suppl):S40-S59

59 Brott TG, Hobson RW II, Howard G, et al; CREST Investigators. Stenting versus endarterectomy for treatment of carotid-artery stenosis. N Engl J Med 2010;363(1):11-23

60 Bates ER, Babb JD, Casey DE, et al. ACCF/SCAI/SVMB/SIR/ ASITN 2007 clinical expert consensus document on carotid stenting: a report of the American College of Cardiology Foundation Task Force on Clinical Expert Consensus Documents (ACCF/SCAI/SVMB/SIR/ASITN Clinical Expert Consensus Document Committee on Carotid Stenting). J Am Coll Cardiol 2007;49:126-170

61 Lakhani S, Guha A, Nahser HC. Anaesthesia for endovascular management of cerebral aneurysms. Eur J Anaesthesiol 2006;23(11):902-913

62 See JJ, Manninen PH. Anesthesia for neuroradiology. Curr Opin Anaesthesiol 2005;18(4):437-441

63 Tepel M, van der Giet M, Schwarzfeld C, Laufer U, Liermann D, Zidek W. Prevention of radiographic-contrast-agent-induced reductions in renal function by acetylcysteine. $\mathrm{N}$ Engl J Med 2000;343(3):180-184

64 Beckett KR, Moriarity AK, Langer JM. Safe use of contrast media: what the radiologist needs to know. Radiographics 2015;35(6):1738-1750

65 The 2007 Recommendations of the International Commission on Radiological Protection. Biological and Epidemiological information on health risks attributable to ionizing radiation. ICRP Publication 103. Ann ICRP 2007;37:137-246

66 Miller DL, Vañó E, Bartal G, et al; Cardiovscular and Interventional Radiology Society of Europe; Society of Interventional Radiology. Occupational radiation protection in interventional radiology: a joint guideline of the Cardiovascular and Interventional Radiology Society of Europe and the Society of Interventional Radiology. Cardiovasc Intervent Radiol 2010;33(2):230-239

67 Hayman MW, Paleologos MS, Kam PC. Interventional neuroradiological procedures-a review for anaesthetists. Anaesth Intensive Care 2013;41(2):184-201

68 Murad MH, Shahrour A, Shah ND, Montori VM, Ricotta JJ. A systematic review and meta-analysis of randomized trials of carotid endarterectomy vs stenting. J Vasc Surg 2011;53(3):792-797

69 Sánchez-Arjona MB, Sanz-Fernández G, Franco-Macías E, Gil-Peralta A. Cerebral hemodynamic changes after carotid angioplasty and stenting. AJNR Am J Neuroradiol 2007;28(4):640-644

70 Dangas G, Laird JR Jr, Satler LF, et al. Postprocedural hypotension after carotid artery stent placement: predictors and short- and long-term clinical outcomes. Radiology 2000;215(3):677-683

71 Chimowitz MI, Lynn MJ, Derdeyn CP, et al; SAMMPRIS Trial Investigators. Stenting versus aggressive medical therapy for intracranial arterial stenosis. $\mathrm{N}$ Engl J Med 2011;365(11):993-1003

72 Lee $\mathrm{IH}$, Kim $\mathrm{KH}$, Jeon P, et al. Ethanol sclerotherapy for the management of craniofacial venous malformations: the interim results. Korean J Radiol 2009;10(3):269-276

73 Powers WJ, Rabinstein AA, Ackerson T, et al. Guidelines for the early management of patients with acute ischemic stroke: a guideline for healthcare professionals from the American Heart Association/American Stroke Association. Stroke 2018;49(3):e46-e110 
74 Bösel J, Hacke W, Bendszus M, Rohde S. Treatment of acute ischemic stroke with clot retrieval devices. Curr Treat Options Cardiovasc Med 2012;14(3):260-272

75 Davis MJ, Menon BK, Baghirzada LB, et al; Calgary Stroke Program. Anesthetic management and outcome in patients during endovascular therapy for acute stroke. Anesthesiology 2012;116(2):396-405

76 Ahmed N, Wahlgren N, Brainin M, et al; SITS Investigators. Relationship of blood pressure, antihypertensive therapy, and outcome in ischemic stroke treated with intravenous thrombolysis: retrospective analysis from Safe Implementation of Thrombolysis in Stroke-International Stroke Thrombolysis Register (SITS-ISTR) Stroke 2009;40(7):2442-2449
77 Saini M, Saqqur M, Kamruzzaman A, Lees KR, Shuaib A; VISTA Investigators. Effect of hyperthermia on prognosis after acute ischemic stroke. Stroke 2009;40(9):3051-3059

78 Armonda RA, Vo AH, Dunford J, Bell RS. Anesthesia for endovascular neurosurgery. Neurosurgery 2006;59(5, Suppl 3):S66-S76, discussion S3-S13

79 Missant C, Van de Velde M. Morbidity and mortality related to anaesthesia outside the operating room. Curr Opin Anaesthesiol 2004;17(4):323-327 